\title{
Interpretation of Near-Infrared Spectroscopy (NIRS) Signals in Skeletal Muscle
}

\author{
Adeola A. Sanni *(D) and Kevin K. McCully \\ Department of Kinesiology, University of Georgia, Athens, GA 30602, USA; mccully@uga.edu \\ * Correspondence: aas56767@uga.edu
}

Received: 5 April 2019; Accepted: 23 May 2019; Published: 26 May 2019

\begin{abstract}
Near-infrared spectroscopy (NIRS) uses the relative absorption of light at 850 and $760 \mathrm{~nm}$ to determine skeletal muscle oxygen saturation. Previous studies have used the ratio of both signals to report muscle oxygen saturation. Purpose: The purpose of this pilot study is to assess the different approaches used to represent muscle oxygen saturation and to evaluate the pulsations of oxygenated hemoglobin/myoglobin $\left(\mathrm{O}_{2}\right.$ heme) and deoxygenated hemoglobin/myoglobin (Heme) signals. Method: Twelve participants, aged 20-29 years, were tested on the forearm flexor muscles using continuous-wave NIRS at rest. Measurements were taken during 2-3 min rest, physiological calibration (5 min ischemia), and reperfusion. Ten participants were included in the study analysis. Results: There was a significant difference in pulse size between $\mathrm{O}_{2}$ heme and Heme signals at the three locations $(p<0.05)$. Resting oxygen saturation was $58.8 \%+9.2 \%, 69.6 \%+3.9 \%$, and $89.2 \%$ $+6.9 \%$ when calibrated using $\mathrm{O}_{2}$ heme, the tissue oxygenation/saturation index (TSI), and Heme, respectively. Conclusion: The difference in magnitude of $\mathrm{O}_{2}$ heme and Heme pulses with each heartbeat might suggest different anatomical locations of these signals, for which calibrating with just one of the signals instead of the ratio of both is proposed. Calculations of physiological calibration must account for increased blood volume in the tissue because of the changes in blood volume, which appear to be primarily from the $\mathrm{O}_{2}$ heme signal. Resting oxygen levels calibrated with Heme agree with theoretical oxygen saturation.
\end{abstract}

Keywords: near-infrared spectroscopy (NIRS); oxygen consumption; hemoglobin; myoglobin; skeletal muscle

\section{Introduction}

Skeletal muscle contractions play an essential role in human locomotion by generating the forces required for movement [1]. A key to muscle contractions is the production of chemical energy (ATP) primarily from oxidative metabolism in muscle mitochondria [2,3]. Changes in oxygen levels in skeletal muscle can provide insights into the function of the skeletal muscle in both healthy and diseased states. Near-infrared spectroscopy (NIRS) has been increasingly used to study oxygen levels in skeletal muscle [4-7]. A search of PubMed in January 2019 for the terms "NIRS" and "muscle" showed 679 publications, 126 of which are from the last two years. NIRS measurements of muscle oxygen level, blood flow, and metabolism are noninvasive and have shown good agreement with other measurement modalities [8]. Continuous-wavelength NIRS devices use physiological calibration (vascular occlusion) to $0 \%$; this has been shown to allow accurate quantitative measurements in the skeletal muscle [4].

Several methodological questions about NIRS measurements of skeletal muscle remain [7,9], such as which of the NIRS signals is the most appropriate to report, and if physiological calibration ( $5 \mathrm{~min}$ ischemia) reaches $0 \%$ oxygen saturation. NIRS measurements typically record absorbance of light at several wavelengths, where changes in absorbance in the region near $850 \mathrm{~nm}$ are ascribed to 
oxygenated hemoglobin/myoglobin $\left(\mathrm{O}_{2}\right.$ heme), and absorbance in the region near $760 \mathrm{~nm}$ is attributed to deoxygenated hemoglobin/myoglobin (Heme). Often, a ratio of absorbance at 850/760 nm or 850/(850 + 760) $\mathrm{nm}$ is used to describe oxygen saturation [10]. A popular ratio is the tissue oxygenation/saturation index (TSI), which is the ratio of absorbance at $850 /(850+760) \mathrm{nm} \times 100$ to produce percentage values [11]. However, some investigators, such as Grassi et al. [7], have proposed using only the Heme signal because it seems to better reflect oxygen extraction in a variety of experimental approaches. Thus, there are still questions regarding the most appropriate NIRS signal to report. There are also questions as to whether the NIRS pulsation signal in skeletal muscle reflects contributions from hemoglobin or myoglobin $[7,12]$. Some investigators have suggested that myoglobin represents $10 \%$ of the NIRS signal [13,14], while others have reported it reflects $80 \%$ of the signal [15]. Davis et al. [9] reported approximately $32 \%$ hemoglobin in the NIRS signal in human skeletal muscle and that this value depends on anatomical and experimental positions.

The purpose of this study was to evaluate NIRS signals from 760 and $850 \mathrm{~nm}$ in response to ischemia and reperfusion in the forearms of healthy young adults. We hypothesized that: (1) blood volume change alters the physiological calibration using the $\mathrm{O}_{2}$ heme signal, producing a lower-than-expected oxygen saturation value; (2) the physiological calibration produces $\mathrm{O}_{2}$ heme values that can be considered zero oxygen saturation; and (3) pulsations in the NIRS signals due to heart rate reflect oxygen saturation specifically from hemoglobin.

\section{Materials and Methods}

\subsection{Participants}

This project involved a convenience sample of University of Georgia students who volunteered as study participants. Twelve subjects (five males, seven females) aged 20-29 years participated in the study. Ten subjects were included in the final analysis, as two participants' data were excluded because the electrical stimulation current did not activate the muscle and increase the metabolic rate as required (at least a fourfold increase). The study was conducted with the approval of the Institutional Review Board at the University of Georgia (Athens, GA, USA). All subjects gave their informed consent for inclusion before participation. The study was conducted in accordance with the Declaration of Helsinki, and the protocol was approved by the Ethics Committee of the University of Georgia (Protocol \#STUDY00004412) on 11 February 2017.

\subsection{Experimental Design}

This pilot study used a single group design. Comparisons were made between three different experimental conditions (rest, ischemia, and reperfusion) performed during one testing session. The protocol consisted of measuring muscle oxygen consumption at rest, during $5 \mathrm{~min}$ of ischemia, and evaluating NIRS signals during reactive hyperemia after the release of ischemia.

\subsection{Experimental Procedures}

The measurement was done on the forearm flexor muscles with the subjects in a supine position. A continuous-wave NIRS device (Portamon, Artinis Medical Systems, Einsteinweg, Netherlands) was placed on the proximal/medial portion of the forearm [16], which was put in place with a nonelastic wrap. Adipose tissue thickness was measured using ultrasound (LOGIQ, GE Healthcare) as previously described [17]. NIRS measurements were digitally recorded throughout the protocol at an acquisition frequency of $10 \mathrm{~Hz}$.

Neuromuscular electrical stimulation was used to increase muscle metabolic rate. Electrodes $(2 \times$ $2 \mathrm{~cm}$ ) were placed proximally and distally to the NIRS device and connected to a commercial electrical stimulation device (RICH-MAR, theratouch 4.7, Version 15, Chattanooga, TN, USA). The muscle was stimulated at $6 \mathrm{~Hz}$. Biphasic square wave pulses (200 $\mu$ s with a $50 \mu$ s interpulse delay) with 
submaximal current levels (25-40 mA) tolerable for each subject were used to activate the muscle to provide an increase in the metabolic rate [16].

Muscle ischemia was produced with complete vascular occlusions with a blood pressure cuff, (Hokanson, Bellevue, WA, USA) placed about $2 \mathrm{~cm}$ above the elbow. The pressure of the cuff was set to $220-260 \mathrm{mmHg}$ using a rapid cuff inflation system (Hokanson, E20 Rapid Cuff Inflator and a 30 gal capacity commercial air compressor).

\subsection{Testing Protocol}

After a rest period of 2-3 min, the blood pressure cuff was inflated for $30 \mathrm{~s}$ to measure the rate of muscle oxygen consumption, and a $2 \mathrm{~min}$ baseline measure was recorded. Electrical stimulation was introduced for $30 \mathrm{~s}$ to activate the muscle and check the rate of increase in muscle oxygen consumption; this helped identify the current level that was sufficient to activate each participant's muscle. Physiological calibration was performed ( $5 \mathrm{~min}$ ischemia). Cuff inflation for $5 \mathrm{~min}$ was preceded by electrical stimulation using the previously identified current to increase metabolic rate and reduce the time needed to reach full ischemia. Electrical stimulation was also done for $30 \mathrm{~s}$ to the end of the ischemia to check if oxygen consumption reached $0 \%$. The cuff was deflated and measurements were taken until the signal reached peak reactive hyperemia. Figure 1 shows the example graph of the protocol.

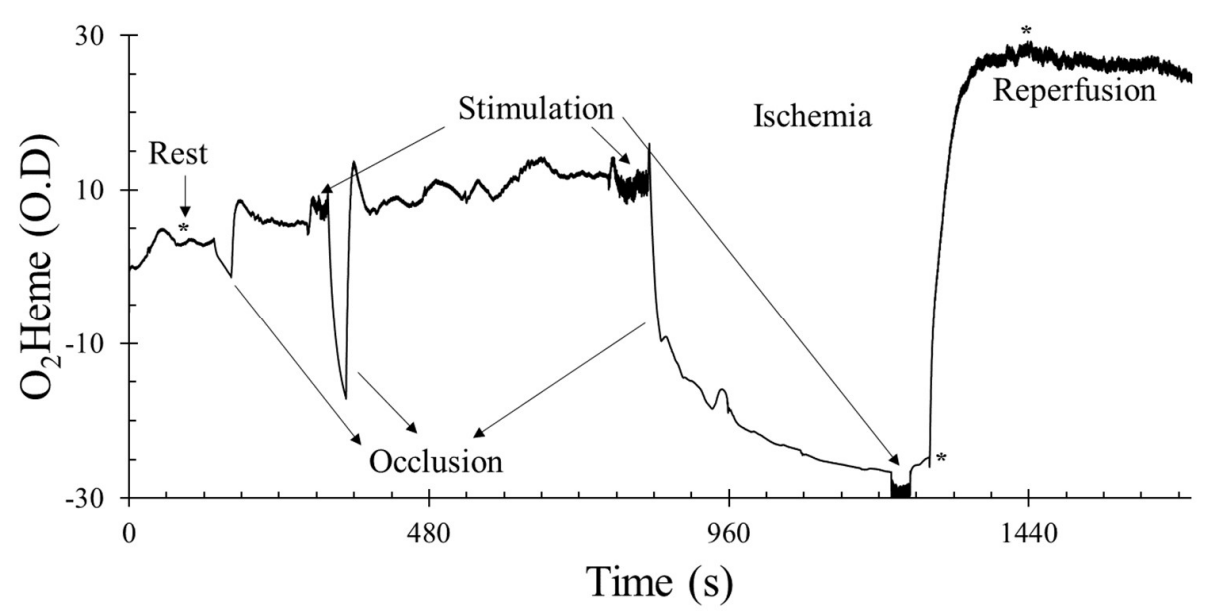

Figure 1. An example of the testing protocol using the oxygenated hemoglobin/myoglobin $\left(\mathrm{O}_{2}\right.$ heme $)$ signal. The $y$-axis scale is in optical density units. * shows the approximate time points when pulse size was calculated at rest, immediately after ischemia, and during reactive hyperemia. $\mathrm{O}_{2}$ heme and deoxygenated hemoglobin/myoglobin (Heme) physiological calibration reached $0 \%$ during ischemia and $100 \%$ at peak hyperemia.

\subsection{Data Analysis}

Oxygen consumption and oxygen delivery were measured using $\mathrm{O}_{2}$ heme and Heme, respectively. The raw data collected from the NIRS device were exported and analyzed on Microsoft Excel. A graph of the NIRS signal (optical density, OD) was plotted against time (calculated using the frequency of data collection). The measurement was recorded from the third channel of the NIRS signal; this was done to avoid the influence of adipose tissue thickness at the shallow channel [18]. OD was recorded at different points of oxygen consumption. The percent pulse size from each NIRS signal was measured and compared at rest immediately after ischemia (early reperfusion) and during peak reactive hyperemia. Pulse size was calculated by finding an average of three consecutive pulsations (wave heights) and subtracting the average of the two minimum pulse signals (troughs) from the maximum pulse signal (crest), multiplied by 100; values are in OD. All measurements were calibrated (physiological calibration) with the delta range of reactive hyperemia after $5 \mathrm{~min}$ of ischemia. Pulse size was also calculated as $32 \%$ of hemoglobin relative to total Heme (hemoglobin plus myoglobin), as recommended by Davis and Barstow [9]. 


\subsection{Statistical Analysis}

Data were analyzed using IBM SPSS Statistics software v24 (IBM®, Armonk, NY, USA). One-way ANOVA was used to identify the difference among values of resting oxygen saturation calculated using $\mathrm{O}_{2}$ heme, Heme, and TSI signals, and a pairwise Bonferroni post hoc comparison was made to evaluate the individual paired difference. A paired sample $t$-test was used to test the difference in oxygen saturation before and after electrical stimulation during the physiological calibration ( $5 \mathrm{~min}$ cuff). A $3 \times 2$ factor ANOVA was used to identify the difference between $\mathrm{O}_{2}$ heme and Heme pulse size at rest immediately after ischemia and during peak reactive hyperemia. The significance level was set at $\leq 0.05$ (two-tailed) for all comparisons with post-hoc adjustments used as needed for multiple comparisons.

\section{Results}

The characteristics of the participants in this study are shown in Table 1. An example protocol used in this study is shown in Figure 1.

Table 1. Characteristics of study participants.

\begin{tabular}{lll}
\hline & Male & Female \\
\hline$N$ & 5 & 5 \\
\hline Age (years) & $23.6(4.3)$ & $20.2(0.25)$ \\
\hline Height $(\mathrm{cm})$ & $170(0.2)$ & $1.66(0.04)$ \\
\hline Weight $(\mathrm{kg})$ & $73.4(12.3)$ & $64.22(10.5)$ \\
\hline Body Mass Index $\left(\mathrm{kg} / \mathrm{m}^{2}\right)$ & $25.2(3.4)$ & $23.51(4.9)$ \\
\hline Adipose Tissue Thickness $(\mathrm{cm})$ & $0.34(0.09)$ & $0.40(0.22)$ \\
\hline \multicolumn{3}{c}{ Values are means (standard deviations). }
\end{tabular}

\subsection{Oxygen Saturation}

Figure 2A shows reactive hyperemia using the Heme and $\mathrm{O}_{2}$ heme signals, including the difference in both signals (blood volume). There was a significant increase in blood volume during reperfusion, which was about $16 \% \pm 6 \%$ in the $\mathrm{O}_{2}$ heme signal. Resting oxygen saturation was calculated using three different methods (Figure 2B). There was a significant difference among the three values $(F(2,27)=48.2$, $p<0.001)$. Pairwise comparisons showed significant individual paired difference between the $\mathrm{O}_{2}$ heme, TSI, and Heme signals ( $p<0.05$ for all comparisons).

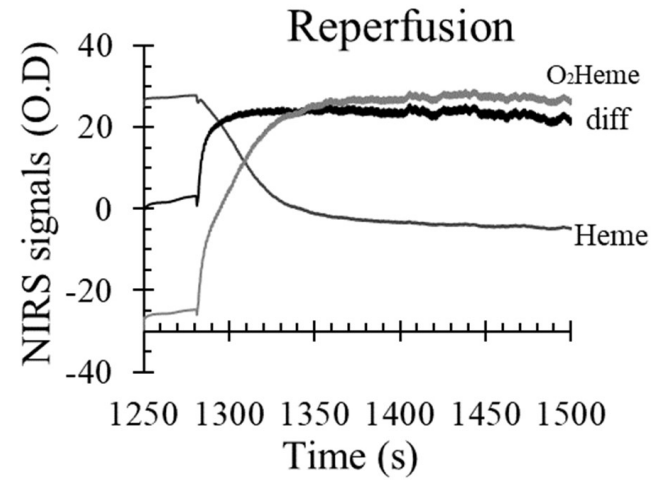

(A)

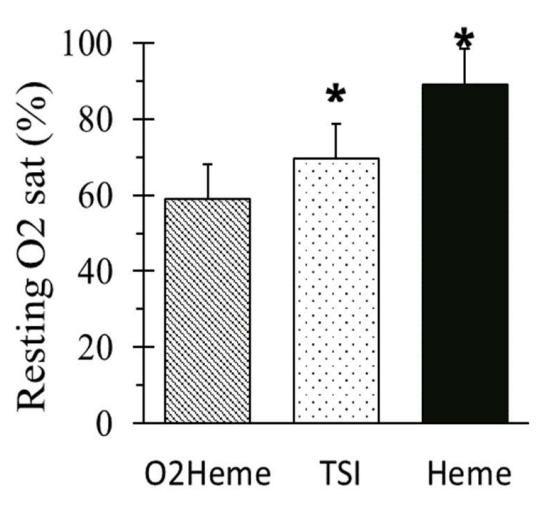

(B)

Figure 2. (A) The $\mathrm{O}_{2}$ heme and Heme signals during reactive hyperemia and the difference in both signals which indicated the influence of blood volume change. (B) The percent of oxygen saturation at rest from three different methods of calculating oxygen saturation $\left(\mathrm{O}_{2}\right.$ heme, tissue oxygenation/saturation index (TSI), and Heme). * indicates a significant difference in the method of calculating oxygen saturation $(p<0.01$ for the three comparisons). Values are means \pm SD. 


\subsection{Physiological Calibration}

With ischemia, the $\mathrm{O}_{2}$ heme signal decreased and the Heme signal increased, showing an increase in deoxygenated blood and a decrease in oxygenated blood due to muscle oxygen consumption. There were no significant changes in either signal with electrical stimulation after 4-5 min of ischemia $\left(\mathrm{O}_{2}\right.$ heme signal, $p=0.148$; Heme signal, $\left.p=0.598\right)$. Figure 3 shows the mean difference and confidence interval of oxygen level before and after stimulation during the $5 \mathrm{~min}$ ischemia. TSI reached approximately the minimum $(45 \% \pm 11 \%)$ and maximum $(76 \% \pm 5 \%)$, respectively.

\section{Difference in Oxygen level During Ischemia}

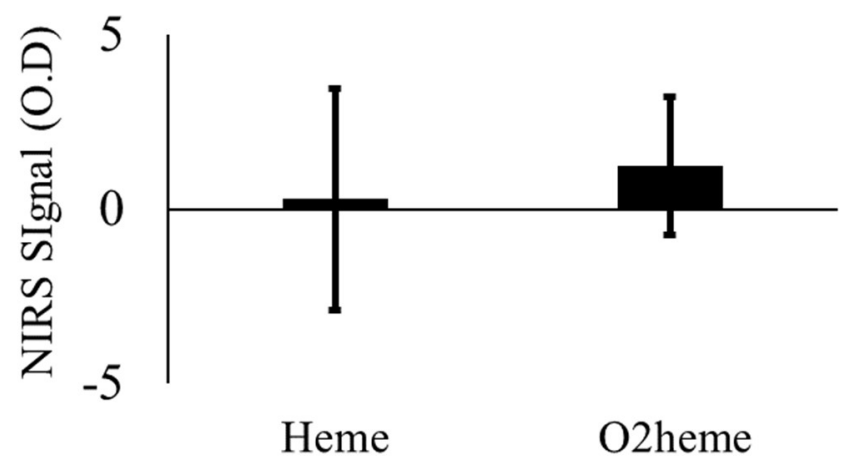

Figure 3. The change in $\mathrm{O}_{2}$ heme and Heme signals before and after stimulation during the 5 min of ischemia with prior stimulation. There was no significant difference, $p>0.05$. Values are means with the $95 \%$ confidence intervals.

\subsection{NIRS Signal Pulsation}

Representative images of heart-rate-induced pulse sizes from both $\mathrm{O}_{2}$ heme and Heme signals are shown in Figure 4. Pulse sizes were calibrated to the ischemic/hyperemia range and the assumed percent of hemoglobin in the NIRS signals [9]. There was a significant interaction between the three locations and the two NIRS signals $\left(F\left(2,36=7.76, \eta^{2}=0.30, p<0.01\right)\right.$. There was a significant difference among the pulse sizes at the three locations (rest, cuff end, and hyperemia) $\left(F(2,36)=75.9, \eta^{2}=0.81, p<0.001\right)$. Bonferroni multiple correction showed a significant difference between each pair of locations $(p<0.001)$. There was a significant main effect difference between $\mathrm{O}_{2}$ heme and Heme pulse size $\left(F\left(1,18=60.9, \eta^{2}=0.77, p<0.001\right)\right.$. Pulse sizes were larger for the $\mathrm{O}_{2}$ heme signal compared with the Heme signal and were largest later during reactive hyperemia compared with during early reperfusion and when at rest (Figure 5A). The ratios of pulse sizes between $\mathrm{O}_{2}$ heme and Heme were not similar to the oxygen saturation values for the NIRS signals at the three locations (Figure 5B). 
$\mathrm{O}_{2}$ Heme rest

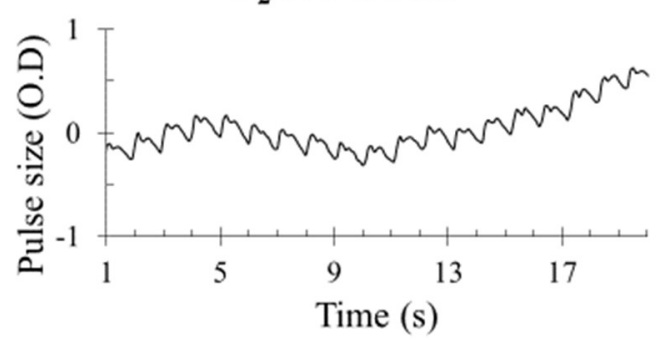

$\mathrm{O}_{2}$ Heme hyperemia

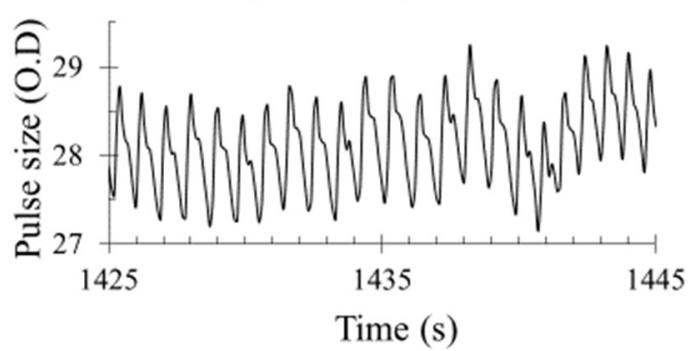

Heme rest

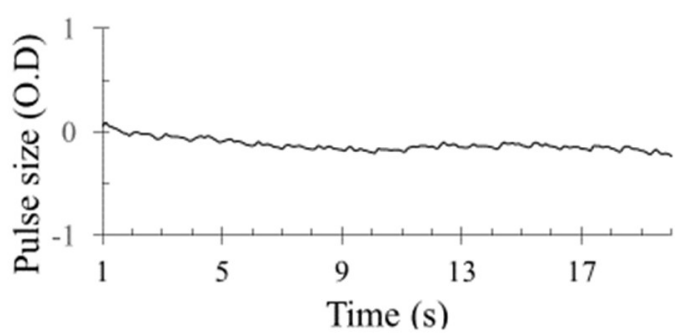

Heme hyperemia

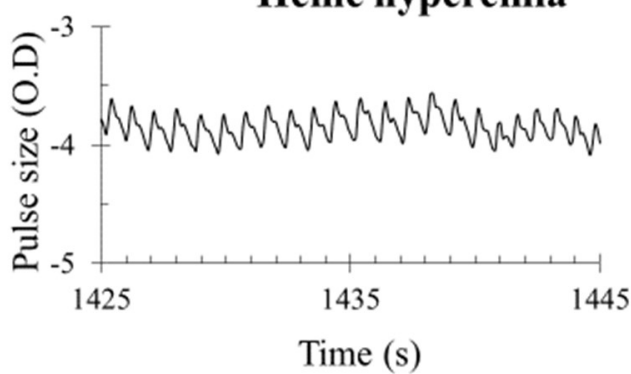

Figure 4. Examples of blood flow pulsations of $\mathrm{O}_{2}$ heme and Heme signals at rest and during peak reactive hyperemia. The $y$-axis scale is in optical density units.

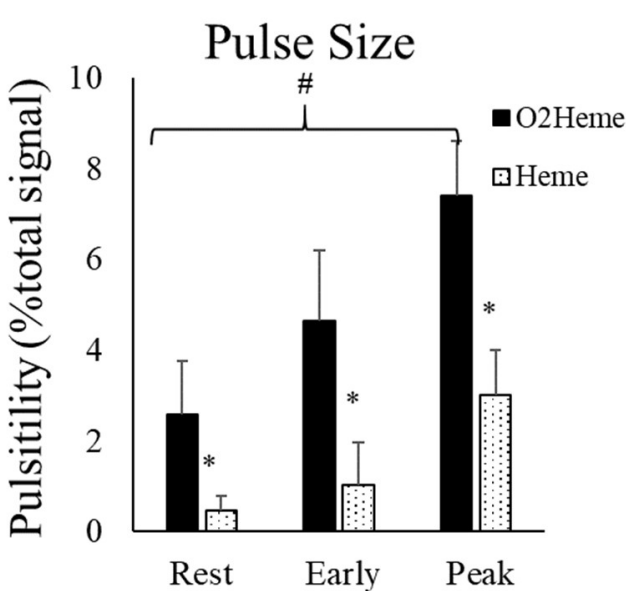

(A)

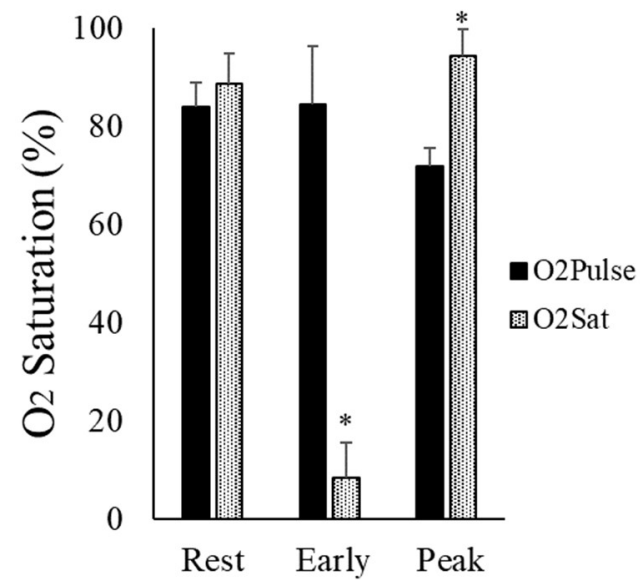

(B)

Figure 5. (A) The average pulse size signals of $\mathrm{O}_{2}$ heme and Heme at rest, immediately after ischemia, and during reactive hyperemia. The $y$-axis scales are in percentage of the calculated physiological calibration. \# indicates a significant difference in the pulse size at each location. * indicates a significant difference between the $\mathrm{O}_{2}$ heme and Heme signals. $p<0.001$ for all comparisons. (B) The ratio of the pulse size at the three different locations and the oxygen saturation calibrated to each signal at the same location. * shows the difference between ratios of pulse and oxygen saturation at each location. Values are means \pm SD.

\section{Discussion}

This study evaluated the use of NIRS signals to determine oxygen levels in skeletal muscle. NIRS signals at 850 and $760 \mathrm{~nm}$ light were presented as $\mathrm{O}_{2}$ heme and Heme to reflect the contribution of myoglobin and hemoglobin. Previous studies have presented NIRS signals as $\mathrm{O}_{2} \mathrm{Hb}$ and $\mathrm{HHb}$, or as $\mathrm{Hb} / \mathrm{Mb}$ [4-7]. This was done based on observations that the NIRS signals come from both hemoglobin and myoglobin and the need to provide more precise terminology for NIRS signals [19]. 


\subsection{Appropriate NIRS Signal for Oxygen Saturation in the Muscle}

This study found that physiological calibration includes a transient increase in blood volume, which appears to be entirely from the $\mathrm{O}_{2}$ heme signal. Calculating a resting oxygen saturation value using $\mathrm{O}_{2}$ heme or a ratio of both $\mathrm{O}_{2}$ heme and Heme (such in TSI) resulted in oxygen saturation values that were lower than values obtained if only the Heme signal was used. The resting oxygen saturation values $(\sim 59 \%)$ found with the $\mathrm{O}_{2}$ heme difference signal was similar to that seen in previous studies $(<70 \%)[20,21]$. However, using only the Heme signal for physiological calibration resulted in resting oxygen saturation values of $91 \%$. This value is consistent with the expected value of Heme oxygen saturation ( $88 \%$ ) based on the assumptions that, at rest, myoglobin oxygen saturation is $100 \%$ [22], the myoglobin contribution to the total Heme in muscle is $70 \%$, and there are hemoglobin saturation values of $70 \%$ (between $98 \%$ in the artery and $40 \%$ in the veins) and a hemoglobin contribution to the total Heme of $30 \%$. For the resting oxygen saturation of the total Heme to be near $60 \%$, either there must be significant myoglobin desaturation at rest or hemoglobin oxygen saturation needs to be less than zero, neither of which is supported in the literature. The use of the deoxygenated signal (Heme) to reflect changes in oxygen levels in muscle has been suggested previously $[7,23,24]$. Interestingly, TSI is a commonly used approach to present oxygen saturation values, and in our study, resting TSI was $70 \%$, which is consistent with previous studies. TSI also does not agree with the calculated Heme oxygen saturations (above). TSI is determined from the ratio of light absorbance at the two wavelengths and never approaches either zero or $100 \%$ oxygen levels during a physiological calibration. Therefore, we propose that oxygen saturation should be calibrated with the (physiological calibration) ischemia protocol [25] using the Heme signal.

\subsection{Physiological Calibration Using 5 Min Ischemia with Prior Exercise}

This study found that $5 \mathrm{~min}$ of ischemia with a prior $30 \mathrm{~s}$ of electrical stimulation resulted in a minimal value of Heme oxygen levels. This was shown by the lack of change in either the $\mathrm{O}_{2}$ heme or the Heme signals after an additional electrical stimulation once the signals had plateaued. Previous studies have not always found that $5 \mathrm{~min}$ of ischemia produced minimal oxygen levels or maximal reactive hyperemia. Five minutes of ischemia has produced an $80-90 \%$ change in oxygen saturation levels [26] and about $80-90 \%$ of the maximal hyperemic blood flow response measured with ultrasound [27]. However, the use of prior exercise to increase metabolic rate (in this study, it produced an increase in metabolic rate approximately fivefold above resting metabolic rate) did appear to result in complete desaturation of the muscle. The prior use of exercise or electrical stimulation to increase metabolic rate has been proposed to assure a maximal change in oxygen levels and blood flow [28].

\subsection{Interpretation of the Pulsatile $\mathrm{O}_{2}$ heme and Heme Signals from Muscle}

We could not accept the hypothesis that pulsations in the NIRS signals reflect the oxygen saturation from the entire hemoglobin signal. Because the pulsations are due to changes in the hemoglobin signal and not the myoglobin signal, the hypothesis was that the ratio of the signal size of the $\mathrm{O}_{2}$ heme and Heme pulsations would reflect hemoglobin oxygen saturation. However, the ratio of the signal size of the $\mathrm{O}_{2}$ heme and Heme pulsations were significantly different from the hemoglobin oxygen saturation values that would be expected during immediate reperfusion at low oxygen levels as well as during reactive hyperemia once oxygen levels were maximal. Pulsations in blood due to beating of the heart are thought to disappear in capillaries and venules [29]; however, more recent studies have found evidence of pulsations in skeletal muscle capillaries [30]. An alternative hypothesis is that the $\mathrm{O}_{2}$ heme and Heme pulsations represent oxygen saturation levels on the arterial side of the microvascular system. The size of the pulses relative to the estimated total hemoglobin concentrations is small, although this increases during reactive hyperemia when vascular tone is decreased. The presence of pulsations in the Heme signal during peak reactive hyperemia suggests that the $100 \%$ value for total Heme from the physiological calibration is actually less than $100 \%$. This is to be expected, as the muscle 
is still consuming oxygen even during the peak reactive hyperemia time period. However, the size of the Heme pulsations is small enough to allow the assumption of $100 \%$ to be close to accurate, and it would be difficult to accurately determine how much different the actual value would be from $100 \%$. Thus, the pulsations in the $\mathrm{O}_{2}$ heme and Heme signals from NIRS most likely come from precapillary arterioles [31], although changes in vascular tone might alter the microvascular area that contributes to the signal [4].

\subsection{Limitations}

This study was performed using one type of NIRS device: the continuous-wavelength "Portamon" from Artinis Ltd. Most continuous-wavelength devices use similar wavelengths and calculations to determine $\mathrm{O}_{2}$ heme and Heme signals. While the results might be expected to be similar for other continuous-wavelength devices, other devices use phase modulation of photon counting to determine both absorption and scattering of light, allowing more accurate calculations of oxygen levels. How these devices determine $\mathrm{O}_{2}$ heme and Heme might be different enough to produce different relationships between the variables. We used an assumption of the relative contribution of myoglobin and hemoglobin in human muscle based on Davis and Barstow [9]. This assumption was used as a general approximation, as hemoglobin levels can vary between people and experimental conditions [9]. We also tested our subjects in a supine position and did not alter body position during the experiment. Studies where the body is in the standing position, there are changes in body position, or the muscle of interest is at a different height than the heart might have different changes in blood volume from our finding. Under those circumstances, the changes in blood volume might not reflect purely $\mathrm{O}_{2}$ heme as they did in our study. This study included a relatively small sample size of 10 healthy young male and female participants. Future studies could include more heterogeneous populations, including those with clinical conditions, so the findings in the study can be generalized to a larger population. Also, to the best of our knowledge, no study has shown any difference in muscle oxygen between sex, but future studies can consider using sex as a biological variable during NIRS analysis.

\section{Conclusions}

Muscle oxygen levels provide an important window into the function of skeletal muscle. Near-infrared spectroscopy has been increasingly used to evaluate skeletal muscle oxygen levels in both health and disease. This study found that NIRS measurements using a physiological calibration consisting of ischemia with prior exercise can determine a range of oxygen levels in muscle that goes from $0-100 \%$. Calculations of a physiological calibration must account for increased blood volume in the tissue due to reactive hyperemia. Because of the changes in blood volume, which appear to be primarily from the $\mathrm{O}_{2}$ heme signal, the Heme signal is perhaps a better signal with which to perform the calibration. Therefore, identifying the most appropriate NIRS signal to report during measurement of skeletal muscle oxygenation will be beneficial for researchers using NIRS and also clinical practitioners who use the device to measure skeletal muscle oxygenation. Finally, NIRS-based signals have pulsations in signal intensity related to heart rate. These pulsations most likely reflect hemoglobin in the atrial side of the microvascular system and not the entire hemoglobin signal. The conclusions of this pilot study should help improve the interpretation and usefulness of NIRS measurements of skeletal muscle.

Author Contributions: Conceptualization, K.K.M.; methodology, A.A.S. and K.K.M.; software, K.K.M.; formal analysis, A.A.S.; investigation, A.A.S.; data curation, A.A.S.; writing-original draft preparation, A.A.S.; writing-review and editing, A.A.S. and K.K.M.; supervision, A.A.S. and K.K.M.; project administration, K.K.M.

Funding: This research received no external funding.

Acknowledgments: We would like to thank Max Sumner and Rebekah Lavender for their assistance throughout the process of data collection for this study.

Conflicts of Interest: One of the authors, Kevin McCully, is the President and Chief Science Officer of Infrared Rx, Inc., a company that develops analysis software related to NIRS measurements. 


\section{References}

1. Trovato, F.M.; Imbesi, R.; Conway, N.; Castrogiovanni, P. Morphological and Functional Aspects of Human Skeletal Muscle. J. Func. Morphol. Kinesiol. 2016, 1, 289-302. [CrossRef]

2. Addabbo, F.; Ratliff, B.; Park, H.C.; Kuo, M.C.; Ungvari, Z.; Ciszar, A.; Krasnikof, F.; Sodhi, K.; Zhang, F.; Nasjletti, A.; et al. The Krebs cycle and mitochondrial mass are early victims of endothelial dysfunction: Proteomic approach. Am. J. Pathol. 2009, 174, 34-43. [CrossRef]

3. Coen, P.M.; Jubrias, S.A.; Distefano, G.; Amati, F.; Mackey, D.C.; Glynn, N.W.; Manini, T.M.; Wohlgemuth, S.E.; Leeuwenburgh, C.; Cummings, S.R. Skeletal muscle mitochondrial energetics are associated with maximal aerobic capacity and walking speed in older adults. J. Gerontol. A Biol. Sci. Med. Sci. 2013, 68, 447-455. [CrossRef] [PubMed]

4. McCully, K.K.; Hamaoka, T. Near-infrared spectroscopy: What can it tell us about oxygen saturation in skeletal muscle? Exerc. Sport Sci. Rev. 2000, 28, 123-127. [PubMed]

5. Hamaoka, T.; McCully, K.K.; Niwayama, M.; Chance, B. The use of muscle near-infrared spectroscopy in sport, health and medical sciences: Recent developments. Philos. Trans. R. Soc. A 2011, 369, 4591-4604. [CrossRef]

6. Ferrari, M.; Muthalib, M.; Quaresima, V. The use of near-infrared spectroscopy in understanding skeletal muscle physiology: Recent developments. Philos. Trans. A Math. Phys. Eng. Sci. 2011, 369, 4577-4590. [CrossRef] [PubMed]

7. Grassi, B.; Quaresima, V. Near-infrared spectroscopy and skeletal muscle oxidative function in vivo in health and disease: A review from an exercise physiology perspective. J. Biomed. Opt. 2016, 21, 091313. [CrossRef] [PubMed]

8. McCully, K.; Iotti, S.; Kendrick, K.; Wang, Z.; Posner, J.D.; Leigh, J., Jr.; Chance, B. Simultaneous in vivo measurements of $\mathrm{HbO}_{2}$ saturation and PCr kinetics after exercise in normal humans. J. Appl. Physiol. 1994, 77, 5-10. [CrossRef] [PubMed]

9. Davis, M.L.; Barstow, T.J. Estimated contribution of hemoglobin and myoglobin to near infrared spectroscopy. Respir. Physiol. Neurobiol. 2013, 186, 180-187. [CrossRef]

10. Hesford, C.M.; Laing, S.J.; Cardinale, M.; Cooper, C.E. Asymmetry of quadriceps muscle oxygenation during elite short-track speed skating. Med. Sci. Sports Exerc. 2012, 44, 501-508. [CrossRef] [PubMed]

11. Jones, B.; Cooper, C.E. Near Infrared Spectroscopy (NIRS) Observation of Vastus Lateralis (Muscle) and Prefrontal Cortex (Brain) Tissue Oxygenation During Synchronised Swimming Routines in Elite Athletes. In Oxygen Transport to Tissue XL; Thews, O., LaManna, J.C., Harrison, D.K., Eds.; Springer: New York, NY, USA, 2018; pp. 111-117.

12. Bendahan, D.; Chatel, B.; Jue, T. Comparative NMR and NIRS analysis of oxygen-dependent metabolism in exercising finger flexor muscles. Am. J. Physiol. Regul. Integr. Comp. Physiol. 2017, 313, R740-R753. [CrossRef] [PubMed]

13. Seiyama, A.; Hazeki, O.; Tamura, M. Noninvasive quantitative analysis of blood oxygenation in rat skeletal muscle. J. Biochem. 1988, 103, 419-424. [CrossRef] [PubMed]

14. Mancini, D.M.; Bolinger, L.; Li, H.; Kendrick, K.; Chance, B.; Wilson, J.R. Validation of near-infrared spectroscopy in humans. J. Appl. Physiol. 1994, 77, 2740-2747. [CrossRef]

15. Marcinek, D.J.; Amara, C.E.; Matz, K.; Conley, K.E.; Schenkman, K.A. Wavelength shift analysis: A simple method to determine the contribution of hemoglobin and myoglobin to in vivo optical spectra. Appl. Spectrosc. 2007, 61, 665-669. [CrossRef]

16. Bossie, H.M.; Willingham, T.B.; Schoick, R.A.V.; O'Connor, P.J.; McCully, K.K. Mitochondrial capacity, muscle endurance, and low energy in friedreich ataxia. Muscle Nerve 2017, 56, 773-779. [CrossRef]

17. Ryan, T.E.; Erickson, M.L.; Brizendine, J.T.; Young, H.J.; McCully, K.K. Noninvasive evaluation of skeletal muscle mitochondrial capacity with near-infrared spectroscopy: Correcting for blood volume changes. J. Appl. Physiol. 2012, 113, 175-183. [CrossRef]

18. Niemeijer, V.M.; Spee, R.F.; Jansen, J.P.; Buskermolen, A.B.; van Dijk, T.; Wijn, P.F.; Kemps, H.M. Test-retest reliability of skeletal muscle oxygenation measurements during submaximal cycling exercise in patients with chronic heart failure. Clin. Physiol. Funct. Imaging 2017, 37, 68-78. [CrossRef] [PubMed]

19. Barstow, T.J. CORP: Understanding near infrared spectroscopy (NIRS) and its application to skeletal muscle research. J. Appl. Physiol. 2019, 126, 1360-1376. [CrossRef] 
20. McCully, K.K. The influence of passive stretch on muscle oxygen saturation. Adv. Exp. Med. Biol. 2010, 662, 317-322.

21. Quaresima, V.; Colier, W.N.; Van Der Sluijs, M.; Ferrari, M. Nonuniform quadriceps O2 consumption revealed by near infrared multipoint measurements. Biochem. Biophys. Res. Commun. 2001, 285, 1034-1039. [CrossRef]

22. Richardson, R.S.; Grassi, B.; Gavin, T.P.; Haseler, L.J.; Tagore, K.; Roca, J.A.; Wagner, P.D. Evidence of O2 supply-dependent VO2 max in the exercise-trained human quadriceps. J. Appl. Physiol. 1999, 86, 1048-1053. [CrossRef] [PubMed]

23. Soares, R.N.; McLay, K.M.; George, M.A.; Murias, J.M. Differences in oxidative metabolism modulation induced by ischemia/reperfusion between trained and untrained individuals assessed by NIRS. Physiol. Rep. 2017, 5, e13384. [CrossRef] [PubMed]

24. MacDonald, M.; Tarnopolsky, M.A.; Green, H.J.; Hughson, R.L. Comparison of femoral blood gases and muscle near-infrared spectroscopy at exercise onset in humans. J. Appl. Physiol. 1999, 86, 687-693. [CrossRef] [PubMed]

25. Southern, W.M.; Ryan, T.E.; Reynolds, M.A.; McCully, K. Reproducibility of near-infrared spectroscopy measurements of oxidative function and postexercise recovery kinetics in the medial gastrocnemius muscle. Appl. Physiol. Nutr. Metab. 2014, 39, 521-529. [CrossRef] [PubMed]

26. Hamaoka, T.; McCully, K.K.; Quaresima, V.; Yamamoto, K.; Chance, B. Near-infrared spectroscopy/imaging for monitoring muscle oxygenation and oxidative metabolism in healthy and diseased humans. J. Biomed. Opt. 2007, 12, 062105. [CrossRef]

27. Stoner, L.; McCully, K.K. Peak and time-integrated shear rates independently predict flow-mediated dilation. J. Clin. Ultrasound 2012, 40, 341-351. [CrossRef] [PubMed]

28. Hamaoka, T.; Iwane, H.; Shimomitsu, T.; Katsumura, T.; Murase, N.; Nishio, S.; Osada, T.; Kurosawa, Y.; Chance, B. Noninvasive measures of oxidative metabolism on working human muscles by near-infrared spectroscopy. J. Appl. Physiol. 1996, 81, 1410-1417. [CrossRef]

29. Folkow, B.; Haglund, U.; Jodal, M.; Lundgren, O. Blood flow in the calf muscle of man during heavy rhythmic exercise. Acta Physiol. Scand. 1971, 81, 157-163. [CrossRef]

30. Lee, J.J.; Tyml, K.; Menkis, A.H.; Novick, R.J.; McKenzie, F.N. Evaluation of pulsatile and nonpulsatile flow in capillaries of goat skeletal muscle using intravital microscopy. Microvasc. Res. 1994, 48, 316-327. [CrossRef]

31. Huo, Y.; Kassab, G.S. Pulsatile blood flow in the entire coronary arterial tree: Theory and experiment. Am. J. Physiol. Heart Circ. Physiol. 2006, 291, H1074-H1087. [CrossRef] 